\title{
DOUBLE SUMS OF KLOOSTERMAN SUMS IN FINITE FIELDS
}

\author{
SIMON MACOURT AND IGOR E. SHPARLINSKI
}

\begin{abstract}
We bound double sums of Kloosterman sums over a finite field $\mathbb{F}_{q}$, with one or both parameters ranging over an affine space over its prime subfield $\mathbb{F}_{p} \subseteq \mathbb{F}_{q}$. These are finite fields analogues of a series of recent results by various authors in finite fields and residue rings. Our results are based on recent advances in additive combinatorics in arbitrary finite field.
\end{abstract}

\section{INTRODUCTION}

1.1. Background. The motivation behind this work comes from recent advances in estimating various bilinear sums of Kloosterman sums which have found a wealth of applications to various arithmetic problems, see $[1,4,6-8,10,11]$ and references therein.

Here we extend some of these results to the settings of finite fields. Our approach is modelled from that of $[10,11]$ however at one significant point it deviates and we employ some very recent results of [9] from additive combinatorics in arbitrary finite fields.

For a prime power $q$, let $\mathbb{F}_{q}$ denote the finite field $\mathbb{K}=\mathbb{F}_{q}$ of $q$ elements.

We fix a nontrivial additive character $\psi$ of $\mathbb{F}_{q}$ and for integers $u, v \in$ $\mathbb{F}_{q}$ we define the Kloosterman sum

$$
\mathcal{K}_{\psi}(u, v)=\sum_{x \in \mathbb{F}_{q}^{*}} \psi\left(u x+v x^{-1}\right) .
$$

We consider sums of Kloosterman sums

$$
\mathcal{S}_{\psi}(\mathcal{U}, \mathcal{V})=\sum_{u \in \mathcal{U}} \sum_{v \in \mathcal{V}} \mathcal{K}_{\psi}(u, v)
$$

Date: March 26, 2019.

2010 Mathematics Subject Classification. 11T23.

Key words and phrases. Kloosterman sums, finite fields, double sum, cancellation. 
over some subsets $\mathcal{U}, \mathcal{V} \subseteq \mathbb{F}_{q}$ and also of more general sums,

$$
\mathcal{S}_{\psi}(\boldsymbol{\alpha} ; \mathcal{U}, \mathcal{V})=\sum_{u \in \mathcal{U}} \sum_{v \in \mathcal{V}} \alpha_{v} \mathcal{K}_{\psi}(u, v)
$$

with a sequence of complex weights $\boldsymbol{\alpha}=\left\{\alpha_{v}\right\}_{v \in \mathcal{V}}$.

By the Weil bound we have

$$
\left|\mathcal{K}_{\psi}(u, v)\right| \leqslant 2 q^{1 / 2},
$$

see [5, Corollary 11.12]. Hence we immediately obtain

$$
\left|\mathcal{S}_{\psi}(\boldsymbol{\alpha} ; \mathcal{U}, \mathcal{V})\right| \leqslant 2 U q^{1 / 2} \sum_{v \in \mathcal{V}}\left|\alpha_{v}\right|
$$

where $U=\# \mathcal{U}$ is the cardinality of $\mathcal{U}$.

We are interested in studying cancellations amongst Kloosterman sums and thus improvements of the trivial bound (1.1). We note that the sums $\mathcal{S}_{\psi}(\mathcal{U}, \mathcal{V})$ and $\mathcal{S}_{\psi}(\boldsymbol{\alpha} ; \mathcal{U}, \mathcal{V})$ are finite field analogues of similar sums studied in $[1,4,6-8,10,11]$ in the settings of prime fields and residue rings. Hence, adopting the model of $\mathbb{F}_{q}$ as

$$
\mathbb{F}_{q} \cong \mathbb{F}_{p}[X] / f(x)
$$

for a prime field $\mathbb{F}_{p}$ and an irreducible over $\mathbb{F}_{p}$ polynomial $f$ (of degree $\operatorname{deg} f=\left[\mathbb{F}_{q}: \mathbb{F}_{p}\right]$ ) we expect that our bounds can be used for similar arithmetic applications in function fields. Since in the above works, the case of averaging over intervals plays a vital role, here we consider the case when one or both of the sets $\mathcal{U}$ and $\mathcal{V}$ is an affine subspace of $\mathbb{F}_{q}$, considered as a vector space over its prime subfield $\mathbb{F}_{p} \subseteq \mathbb{F}_{q}$. More precisely, we consider the sums

- $\mathcal{S}_{\psi}(\mathcal{A}, \mathcal{B})$ with two affine spaces $\mathcal{A}$ and $\mathcal{B}$

- $\mathcal{S}_{\psi}(\boldsymbol{\alpha} ; \mathcal{A}, \mathcal{V})$ with an affine space $\mathcal{A}$ and an arbitrary set $\mathcal{V}$.

Our approach is similar to that of $[10,11]$ however some ingredients used in $[10,11]$ are either unknown or do not exist in function field settings. Hence we use a different approach based on additive combinatorics and in particular we rely on recent results of Mohammadi [9].

1.2. Our results. We recall that the notations $U=O(V), U \ll V$ and $V \gg U$ are all equivalent to the statement that the inequality $|U| \leqslant c V$ holds with some constant $c>0$, which is absolute throughout this paper.

We start with the sums $\mathcal{S}_{\psi}(\mathcal{A}, \mathcal{B})$.

Theorem 1.1. Assume that $q=p^{2 k+1}$ is an odd power of a prime $p$. Let $\mathcal{A}$ and $\mathcal{B}$ be affine subspaces of $\mathbb{F}_{q}$ of cardinalities $A=\# \mathcal{A}$ and 
$B=\# \mathcal{B}$, respectively, with. $A \leqslant B$. Then

$$
\mathcal{S}_{\psi}(\mathcal{A}, \mathcal{B}) \ll A B \max \left\{q^{52 / 153},(q / A)^{831 / 832},(q / A)^{761 / 760} q^{-1 / 760}\right\} .
$$

Clearly Theorem (1.1) is only non-trivial for $A \geqslant q^{415 / 831}$ as otherwise the bound $\left|\mathcal{S}_{\psi}(\mathcal{A}, \mathcal{B})\right| \leqslant 2 A B q^{1 / 2}$, implied by (1.1), is stronger.

Given a sequence of complex weights $\boldsymbol{\alpha}=\left\{\alpha_{v}\right\}_{v \in \mathcal{V}}$ supported on a set $\mathcal{V}$ and $\rho>0$, as usual, we define

$$
\|\boldsymbol{\alpha}\|_{\rho}=\left(\sum_{v \in \mathcal{V}}\left|\alpha_{v}\right|^{\rho}\right)^{1 / \rho} .
$$

Theorem 1.2. Assume that $q=p^{2 k+1}$ is an odd power of a prime $p$. Let $\mathcal{A}$ be an affine subspace of $\mathbb{F}_{q}$ of cardinality $A=\# \mathcal{A}$ and let $\mathcal{V} \subseteq \mathbb{F}_{q}$ be an arbitrary set of cardinality $V=\# \mathcal{V}$. Then, for any sequence of complex weights $\boldsymbol{\alpha}=\left\{\alpha_{v}\right\}_{v \in \mathcal{V}}$ we have

$$
\begin{aligned}
& \mathcal{S}_{\psi}(\boldsymbol{\alpha} ; \mathcal{A}, \mathcal{V}) \ll A q^{1 / 4} \sqrt{|\boldsymbol{\alpha}|_{1}|\boldsymbol{\alpha}|_{2}} \\
& \max \left\{q^{13 / 51},\left(\frac{q}{A}\right)^{935 / 1248}, q^{-1 / 1140}\left(\frac{q}{A}\right)^{214 / 285}\right\} .
\end{aligned}
$$

If we suppose that $\left|\alpha_{v}\right| \ll 1$ for all $v \in \mathcal{V}$, then clearly Theorem 1.2 is only non-trivial for $A^{935 / 623} V^{312 / 623}>q$. If we suppose $V=A$ then we have Theorem 1.2 is non-trivial provided $A>q^{623 / 1247}$.

\section{BACKGROUND FROM ADDITIVE COMBINATORICS}

For a set $\mathcal{S} \subseteq \mathbb{F}_{q}$, we use $E(\mathcal{S})$ to denote its additive energy, that is, the number of solutions to the equation

$$
s_{1}+s_{2}=s_{3}+s_{4}, \quad s_{1}, s_{2}, s_{3}, s_{4} \in \mathcal{S} .
$$

Also, as usual, we denote

$$
\mathcal{S}^{-1}=\left\{s^{-1}: s \in \mathcal{S}\right\} \quad \text { and } \quad 2 \mathcal{S}=\{s+t: s+t \in \mathcal{S}\} .
$$

Then by [9, Corollary 5] we have

Lemma 2.1. Let $\mathcal{S} \subseteq \mathbb{F}_{q}$ with

$$
\# \mathcal{S}=S \quad \text { and } \quad \#(2 \mathcal{S})=T
$$

and such that $T \geqslant(\# G)^{52 / 51}$ for any proper subfield $\mathbb{G}$ of $\mathbb{F}_{q}$. Then

$$
E\left(\mathcal{S}^{-1}\right) \ll\left(T^{173 / 104}+q^{-1 / 285} T^{476 / 285}\right) S^{4 / 3} .
$$

It is easy to see that the Cauchy inequality implies the well-known inequality

$$
S^{4} \leqslant \#\left(2 \mathcal{S}^{-1}\right) E\left(\mathcal{S}^{-1}\right) .
$$


Hence from Lemma 2.1 we derive the following (see also [9, Corollary 5]).

Corollary 2.2. Let $\mathcal{S} \subseteq \mathbb{F}_{q}$ with

$$
\# \mathcal{S}=S, \quad \#(2 \mathcal{S})=T, \quad \#\left(2 \mathcal{S}^{-1}\right)=U
$$

and such that $T \geqslant(\# G)^{52 / 51}$ for any proper subfield $\mathbb{G}$ of $\mathbb{F}_{q}$. Then

$$
\max \{T, U\} \gg \min \left\{S^{832 / 831}, q^{1 / 761} S^{760 / 761}\right\} .
$$

\section{Proof of Theorem 1.1}

Changing the order of summation, we obtain

$$
\mathcal{S}_{\psi}(\mathcal{A}, \mathcal{B})=\sum_{x \in \mathbb{F}_{q}^{*}} \sum_{a \in \mathcal{A}} \psi(a x) \sum_{b \in \mathcal{B}} \psi\left(b x^{-1}\right) .
$$

Clearly if $\mathcal{A}$ is a translate of a linear space $\mathcal{L}$ then

$$
\sum_{a \in \mathcal{A}} \psi(a x)= \begin{cases}A, & \text { if } x \in \mathcal{L}^{\perp}, \\ 0, & \text { otherwise }\end{cases}
$$

where $\mathcal{L}^{\perp}$ denotes the the orthogonal complement to $\mathcal{L}$.

Similarly if $\mathcal{B}$ is a translate of a linear space $\mathcal{M}$ then

$$
\sum_{b \in \mathcal{B}} \psi\left(b x^{-1}\right)= \begin{cases}B, & \text { if } x^{-1} \in \mathcal{M}^{\perp}, \\ 0, & \text { otherwise. }\end{cases}
$$

Hence, substituting (3.2) and (3.3) in (3.1), we obtain

$$
\mathcal{S}_{\psi}(\mathcal{A}, \mathcal{B}) \ll A B S
$$

where $S=\# \mathcal{S}$ and the set $\mathcal{S}$ is defined as follows

$$
\mathcal{S}=\left\{x \in \mathbb{F}_{q}^{*}: x \in \mathcal{L}^{\perp} \text { and } x^{-1} \in \mathcal{M}^{\perp}\right\} .
$$

If $S \leqslant q^{52 / 153}$ the result follows immediately. Otherwise we see that

$$
\#(2 \mathcal{S}) \geqslant \# \mathcal{S} \geqslant(\# \mathbb{G})^{52 / 51}
$$

for any proper subfield $\mathbb{G}$ of $\mathbb{F}_{q}$ (since $q$ is not a perfect square we have $\left.\# \mathbb{G} \leqslant q^{1 / 3}\right)$. Hence Corollary 2.2 applies to $\mathcal{S}$.

Since $\mathcal{L}^{\perp}$ and $\mathcal{M}^{\perp}$ are linear spaces, we obviously have

$$
2 \mathcal{S} \subseteq \mathcal{L}^{\perp} \quad \text { and } \quad 2 \mathcal{S}^{-1} \subseteq \mathcal{M}^{\perp} .
$$

Consequently,

$$
\#(2 \mathcal{S}) \leqslant \# \mathcal{L}^{\perp}=q / A \quad \text { and } \quad \#\left(2 \mathcal{S}^{-1}\right) \leqslant \# \mathcal{M}^{\perp}=q / B .
$$

Invoking Corollary 2.2, we obtain

$$
\min \left\{S^{832 / 831}, q^{1 / 761} S^{760 / 761}\right\} \ll \max \{q / A, q / B\} \leqslant q / A
$$


as $A \leqslant B$. Thus

$$
S \ll \max \left\{(q / A)^{831 / 832},(q / A)^{761 / 760} q^{-1 / 760}\right\}
$$

which after substitution in (3.4) implies the result.

\section{Proof of Theorem 1.2}

By changing the order of summation we have

$$
\left|\mathcal{S}_{\psi}(\boldsymbol{\alpha} ; \mathcal{A}, \mathcal{V})\right|=\left|\sum_{x \in \mathbb{F}_{q}^{*}} \sum_{v \in \mathcal{V}} \alpha_{v} \psi\left(v x^{-1}\right) \sum_{a \in \mathcal{A}} \psi(a x)\right|
$$

As previously, if $\mathcal{A}$ is a translate of a linear space $\mathcal{L}$ then

$$
\sum_{a \in \mathcal{A}} \psi(a x)= \begin{cases}A, & \text { if } x \in \mathcal{L}^{\perp} \\ 0, & \text { otherwise }\end{cases}
$$

where $\mathcal{L}^{\perp}$ denotes the orthogonal complement $\mathcal{L}$. It follows that

$$
\begin{aligned}
\left|\mathcal{S}_{\psi}(\boldsymbol{\alpha} ; \mathcal{A}, \mathcal{V})\right| & =A\left|\sum_{x \in \mathcal{L}^{\perp}} \sum_{v \in \mathcal{V}} \alpha_{v} \psi\left(v x^{-1}\right)\right| \\
& \leqslant A\left|\sum_{v \in \mathcal{V}} \alpha_{v}\right|\left|\sum_{x \in \mathcal{L}^{\perp}} \psi\left(v x^{-1}\right)\right| \\
& \leqslant A \sum_{v \in \mathcal{V}}\left|\alpha_{v}\right|^{1 / 2}\left|\alpha_{v}^{2}\right|^{\frac{1}{4}}\left|\sum_{x \in \mathcal{L}^{\perp}} \psi\left(v x^{-1}\right)\right| .
\end{aligned}
$$

Applying the Cauchy-Schwartz inequality twice, we obtain

$$
\begin{aligned}
\left|\mathcal{S}_{\psi}(\boldsymbol{\alpha} ; \mathcal{A}, \mathcal{V})\right|^{4} \leqslant A^{4}\left(\sum_{v \in \mathcal{V}}\left|\alpha_{v}\right|\right)^{2} \sum_{v \in \mathcal{V}}\left|\alpha_{v}\right|^{2} \sum_{v \in \mathcal{V}}\left|\sum_{x \in \mathcal{L}^{\perp}} \psi\left(v x^{-1}\right)\right|^{4} \\
\leqslant A^{4}|\boldsymbol{\alpha}|_{1}^{2}|\boldsymbol{\alpha}|_{2}^{2} \\
\sum_{v \in \mathbb{F}_{q}^{*}} \sum_{w, x, y, z \in \mathcal{L}^{\perp}} \psi\left(v\left(w^{-1}+x^{-1}-y^{-1}-z^{-1}\right)\right) .
\end{aligned}
$$

If $2 \mathcal{L}^{\perp}=\mathcal{L}^{\perp}<(\# G)^{52 / 51}$, we use the trivial bound on additive energy, that is $E\left(\left(\mathcal{L}^{\perp}\right)^{-1}\right) \leqslant\left(\# \mathcal{L}^{\perp}\right)^{3}$, and the result follows immediately. 
Otherwise, we apply Lemma 2.1, observing $\# L^{\perp}=q / A$, to obtain

$$
\begin{gathered}
\mathcal{S}_{\psi}(\boldsymbol{\alpha} ; \mathcal{A}, \mathcal{V}) \ll A q^{1 / 4} \sqrt{|\boldsymbol{\alpha}|_{1}|\boldsymbol{\alpha}|_{2}} \\
\left(\left(\left(\frac{q}{A}\right)^{173 / 104}+q^{-1 / 285}\left(\frac{q}{A}\right)^{476 / 285}\right)\left(\frac{q}{A}\right)^{4 / 3}\right)^{1 / 4} \\
\ll A q^{1 / 4} \sqrt{|\boldsymbol{\alpha}|_{1}|\boldsymbol{\alpha}|_{2}} \\
\max \left\{\left(\frac{q}{A}\right)^{935 / 1248}, q^{-1 / 1140}\left(\frac{q}{A}\right)^{214 / 285}\right\} .
\end{gathered}
$$

This completes the proof.

\section{Comments}

Some of the motivation to this paper comes from an intention to obtain function field analogues of the asymptotic formulas, with a power saving, from $[1,2,10,12]$ for 4 th moments of $L$-functions. However, despite recent progress in this direction due to Florea [3], some ingredients, used in the groundbreaking work of Young [12], remain missing in the function field case.

Finally, we need to impose the condition on $q=p^{2 k+1}$ to avoid th existence of large subfields. It is certainly interesting to drop this restriction and extend our results to even degree extensions.

\section{ACKNOWLEDGEMENTS}

The authors are grateful to Goran Djanković for very illuminating discussions.

This work was supported in part by ARC Grant DP170100786.

\section{REFERENCES}

[1] V. Blomer, É. Fouvry, E. Kowalski, P. Michel and D. Milićević, 'On moments of twisted L-functions', Amer. J. Math. 139 (2017), 707-768. 1, 2, 6

[2] V. Blomer, É. Fouvry, E. Kowalski, P. Michel and D. Milićević, 'Some applications of smooth bilinear forms with Kloosterman sums', Trudy Matem. Inst. Steklov 296 (2017), 24-35. 6

[3] A. Florea, 'The fourth moment of quadratic Dirichlet $L$-functions over function fields', Geom. Funct. Anal. 27 (2017), 541-595. 6

[4] É. Fouvry, E. Kowalski and P. Michel, 'Algebraic trace functions over the primes', Duke Math. J. 163 (2014), 1683-1736. 1, 2

[5] H. Iwaniec and E. Kowalski, Analytic number theory, Amer. Math. Soc., Providence, RI, 2004. 2

[6] K. Liu, I. E. Shparlinski and T. P. Zhang, 'Divisor problem in arithmetic progressions modulo a prime power', Adv. Math. 325 (2018), 459-481. 1, 2

[7] E. Kowalski, P. Michel and W. Sawin, 'Bilinear forms with Kloosterman sums and applications', Ann. Math. 186 (2017), 413-500. 1, 2 
[8] E. Kowalski, P. Michel and W. Sawin, 'Bilinear forms with generalized Kloosterman sums', Preprint, 2018 (available from http://arxiv.org/abs/1802.09849). 1, 2

[9] A. Mohammadi, 'Szemerédi-Trotter type results in arbitrary finite fields', Preprint, 2018 (available from http://arxiv.org/abs/1808.05543). 1, 2, 3, 4

[10] I. E. Shparlinski, 'On sums of Kloosterman and Gauss sums', Trans. Amer. Math. Soc., (to appear). 1, 2, 6

[11] I. E. Shparlinski and T. P. Zhang, 'Cancellations amongst Kloosterman sums', Acta Arith., 176 (2016), 201-210. 1, 2

[12] M. Young, 'The fourth moment of Dirichlet L-functions', Annals Math., 173 (2011), 1-50. 6

Department of Pure Mathematics, University of New South Wales, Sydney, NSW 2052, Australia

E-mail address: s.macourt@unsw.edu.au

School of Mathematics and Statistics, University of New South Wales, Sydney, NSW 2052, Australia

E-mail address: igor.shparlinski@unsw.edu.au 